\title{
Algunas consideraciones sobre el manejo poscosecha de la zanahoria
}

Some considerations on the postharvest management of carrot

\author{
Abel Verdugo-Fuentes ${ }^{1}$ (D), Ofelda Peñuelas-Rubio ${ }^{2}$ (D) Leandris Argentel-Martínez ${ }^{2^{*}}$ (D), \\ José Aurelio Leyva Ponce ${ }^{2}$ (D) , Jorge González-Aguilera ${ }^{3}(\mathrm{D}$
}

\footnotetext{
${ }^{1}$ Centro de Investigación en Alimentación y Desarrollo A. C., Carretera a la Victoria km 0.5, Ejido La Victoria, A.P. 1735, 83000, Hermosillo, Sonora, México.

${ }^{2}$ Tecnológico Nacional de México/Instituto Tecnológico del Valle del Yaqui, Avenida Tecnológico, Block 611, 85260, Bácum, Sonora, México.

${ }^{3}$ Universidade Federal de Mato Grosso do Sul (UFMS), Campus Chapadão do Sul, Caixa Postal 112, 79560-000, Chapadão do Sul/MS, Brasil.
}

*Autor de correspondencia: oleinismora@gmail.com

Fecha de recepción:

4 de septiembre de 2020

Fecha de aceptación:

6 de febrero de 2021

Disponible en línea:

14 de mayo de 2021

Este es un artículo en acceso

abierto que se distribuye de

acuerdo a los términos de la

licencia Creative Commons.

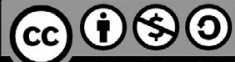

Reconocimiento-

NoComercia-

CompartirIgual 4.0

Internacional

\section{RESUMEN}

La zanahoria posee un alto contenido de carbohidratos, fibra, antioxidantes y minerales y puede consumirse en fresco o procesada. El buen manejo poscosecha permite mantener sus características químicas y propiedades organolépticas idóneas para su consumo. En la presente revisión se aglutinó información sobre el manejo poscosecha de esta especie, enfatizando en los principales factores que afectan su vida de anaquel. La alta tasa de respiración y transpiración, daños mecánicos y mal manejo de las atmósferas de conservación merman su calidad poscosecha.

\section{PALABRAS CLAVE}

Daucus carota, empaque, anaquel, hortalizas.

\section{ABSTRACT}

Carrot has a high content of carbohydrates, fiber, antioxidants, and minerals, and it can be consumed fresh or processed. Good postharvest management allows maintaining its chemical characteristics and organoleptic properties suitable for consumption. This review compiles information on the postharvest management of this species, emphasizing the main factors that affect its shelf life. High respiration and perspiration rate, mechanical damage, and inadequate management of conservation atmospheres reduce its postharvest quality.

\section{KEYWORDS}

Daucus carota, packaging, shelf, vegetables. 


\section{INTRODUCCIÓN}

La zanahoria (Daucus carota L.) pertenece a la familia de las umbelíferas (Hernández y Blanco 2015). Es originaria de Asia Central, particularmente de Afganistán, y fue traída al continente americano por los españoles, para convertirse en un vegetal muy popular (Stolarczyk y Janick 2011). Aunque México no se encuentra entre los tres principales productores (China, Rusia y Estados Unidos) (CEDRSSA 2020), sí aparece como uno de los diez primeros países productores de esta hortaliza en el mundo (Acevedo 2018).

En México, la zanahoria se cultiva en 25 estados; los principales productores son: Zacatecas, Guanajuato, Puebla, Estado de México y Querétaro. De la producción nacional, 88 por ciento se destina al consumo en fresco como mayor preferencia, aunque es significativa la cantidad que se consume procesada (12\%) (SIAP 2018). A escala nacional, en los últimos años se ha incrementado el consumo en forma fresca (zanahorias mínimamente procesadas: lavado, pelado y cortado previo a la venta) (Nesha y Siddiqui 2013). El incremento del consumo se debe a las propiedades químicas que esta hortaliza posee, entre las cuales se destaca su alto contenido de minerales, fibras, carbohidratos antioxidantes como hidroquinonas, catecol, acetato y 2-hidroxiacetofenona (Dong et al. 2021).

Diferentes estudios epidemiológicos han demostrado que el consumo de hortalizas - particularmente de la zanahoria - tiene un efecto benéfico en la salud del consumidor (Rodríguez-Casado 2016) y contribuye a la prevención de procesos degenerativos como la arteriosclerosis y diferentes tipos de cáncer (Turati et al. 2015). El consumo alto de este vegetal implica la necesidad de mantener por más tiempo la mayor calidad, por lo que el productor debe conservar su producto en las mejores condiciones, pues este vegetal es susceptible a diferentes desórdenes morfológicos y fisiológicos como: tamaño no deseado, falta de consistencia del tejido que lo conforma, podredumbres, decoloración y deshidratación (Pandey et al. 2020). Existen, además, algunos trastornos producidos por hongos y bacterias que causan disminución del rendimiento agrícola y, por consiguiente, de su precio, al no cumplir con estándares de calidad (Singh y Sharma 2018). Por ello, es de suma importancia el manejo adecuado poscosecha, utilizando diferentes tecnologías de conservación y empaque, así como la aplicación de diferentes procesos para prolongar su vida de anaquel (Yildiz y Wiley 2017).

El presente trabajo de revisión tiene como objetivo recopilar información científica sobre los principales estudios realizados en el mundo $y$, particularmente, en México, relacionados con el manejo poscosecha de la zanahoria, por lo que puede ser utilizado como material de consulta para contribuir a prolongar su calidad durante el traslado y la venta.

\section{Desarrollo}

\section{Recopilación de la información científica.}

Se llevó a cabo una búsqueda de artículos científicos, libros y folletos en bases de datos como Science Direct, Scielo, Redalyc, Wiley y Google Académico, sobre las principales investigaciones desarrolladas en el mundo relacionadas con el cultivo de la zanahoria en los últimos diez años. Se analizaron los principales trabajos relacionados con los volúmenes de producción, fisiología poscosecha, normas de calidad, desórdenes fisiológicos y patológicos durante el manejo poscosecha, métodos de conservación y tipos de recubrimientos de este cultivo. Finalmente, se hizo una valoración crítica de los estudios relacionados con el manejo poscosecha, principalmente durante el traslado y empaque, para mantener su apariencia física y sus propiedades organolépticas durante la permanencia en anaqueles. Se consultaron 65 fuentes de información, de las cuales, 74 por ciento (48) fueron artículos que tratan temas sobre aspectos relacionados con la poscosecha de esta hortaliza; 10 por ciento (3) corresponden a libros, y 16 por ciento (13) a páginas oficiales que ofrecen elementos estadísticos de superficie sembrada, producción, rendimiento, así como comercialización y estándares de calidad. Paralelamente, se visitaron dos empaques con fines de mercado nacional y exportación, y dos puntos de venta de consumo nacional, para mostrar las características deseadas, los daños mecánicos y los desórdenes fisiológicos que tienen lugar durante el manejo poscosecha de la zanahoria. 


\section{Producción nacional}

La zanahoria ocupa el sexto lugar entre las hortalizas con mayor producción en México, gracias a lo cual el país ocupa el noveno lugar a escala mundial con $366,000 \mathrm{t}$ anuales (Cuadro 1) (CEDRSSA 2020). Por la importancia de esta especie, múltiples estados de la República Mexicana presentan producciones significativas destinadas al consumo nacional y para la exportación.

\section{Fisiología poscosecha}

La zanahoria, como otros vegetales, es un producto perecedero. Entre algunas de las enormes pérdidas que se dan desde su cosecha hasta que llega al consumidor están las que suceden durante los procesos de almacenamiento y comercialización, donde se afecta sobre todo la calidad del producto cosechado. Lo anterior se debe a los desórdenes fisiológicos poscosecha, dentro de los cuales influye

\section{Cuadro 1. Estados de la República Mexicana con mayor superficie dedicada al cultivo de zanahoria y su} rendimiento en 2019.

\begin{tabular}{ccc}
\hline Estado & Superficie sembrada (ha) & Rendimiento t ha $^{-1}$ \\
\hline Guanajuato & 1,716 & 29.2 \\
Puebla & 811 & 31.5 \\
Querétaro & 893 & 32.6 \\
Zacatecas & 707 & 33.9 \\
Sonora & 338 & 26.5 \\
Sinaloa & 200 & 28.0 \\
Baja California Sur & 150 & 20.0 \\
Aguascalientes & 127 & 34.6 \\
\hline
\end{tabular}

Fuente: CEDRSSA (2020).

El rendimiento promedio de los ocho principales productores de zanahoria en México es de $29.5 \mathrm{t}$ ha $^{-1}$ en el ciclo otoño-invierno (CEDRSSA 2020). El principal uso es para consumo en fresco. En la mayoría de los centros de comercialización en el país, el manejo poscosecha es a granel en los anaqueles; para el comercio de exportación se utilizan empaques (Figura 1). Por esta razón, deben conocerse a detalle los aspectos fisiológicos, bioquímicos y microbiológicos que pueden afectar la calidad del producto poscosecha.
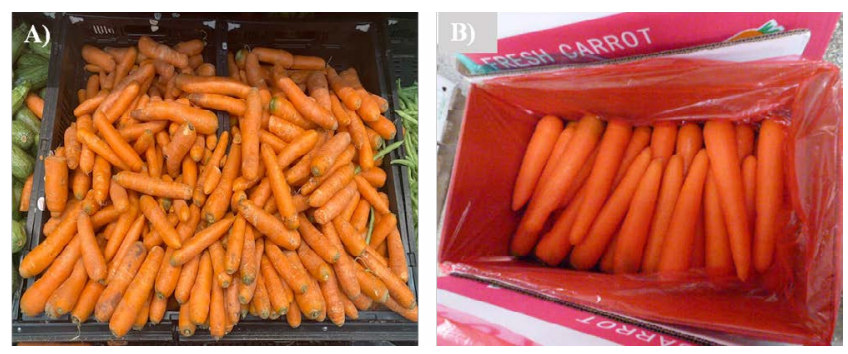

Figura 1. Manejo poscosecha para el consumo nacional (A) y para la exportación (B). la respiración, transpiración y producción de etileno (Firpo et al. 2011).

\section{Respiración}

La intensidad de la respiración constituye un índice de la actividad metabólica de tejidos, aspecto que permite calcular o estimar la duración de su vida útil en función del potencial de deterioro aerobio de los vegetales (Martínez-González et al. 2017). En zanahoria, que normalmente tiene una tasa de respiración alta, se ha demostrado que el manejo poscosecha con técnicas de acondicionamiento - como el uso de recubrimientos comestibles y tratamientos térmicos - reducen significativamente la intensidad de respiración en $11 \mathrm{y}$ 44 por ciento, respectivamente (Escobar et al. 2014). Tales prácticas de conservación pueden contribuir a que se mantenga la apariencia física y turgencia durante el almacenamiento. La temperatura tiene un papel importante en la estabilidad de la respiración (Cuadro 2). Temperaturas más bajas, sin alcanzar la congelación, propician menor tasa respiratoria, lo que 
Cuadro 2. Tasa de respiración de la zanahoria a diferentes temperaturas durante el almacenamiento.

\begin{tabular}{ccc}
\hline Temperatura $\left({ }^{\circ} \mathrm{C}\right)$ & Respiración $\left(\mathrm{mL} \mathrm{CO}_{2} \mathrm{~kg}^{-1} \mathrm{~h}^{-1}\right)$ \\
\hline 0 & $10-20$ \\
5 & $13-26$ \\
10 & $20-42$ \\
15 & $26-54$ \\
20 & $46-95$ \\
\hline
\end{tabular}

Fuente: González et al. (2010).

implica mayor durabilidad del producto, sin perder la apariencia turgente (González et al. 2010).

\section{Transpiración}

La transpiración es una de las causas de deterioro poscosecha de la zanahoria, pues, debido a que el principal componente de esta raíz es agua (86-89\%), la deshidratación ocasiona flacidez (Barzee et al. 2019) (Figura2), aspectoqueresultapocoatractivoydisminuye la calidad comercial (Karnjanawipagul et al. 2010). Considerando que la transpiración se puede reducir disminuyendo la temperatura, aumentando la humedad relativa, y también con la aplicación de un empaque o

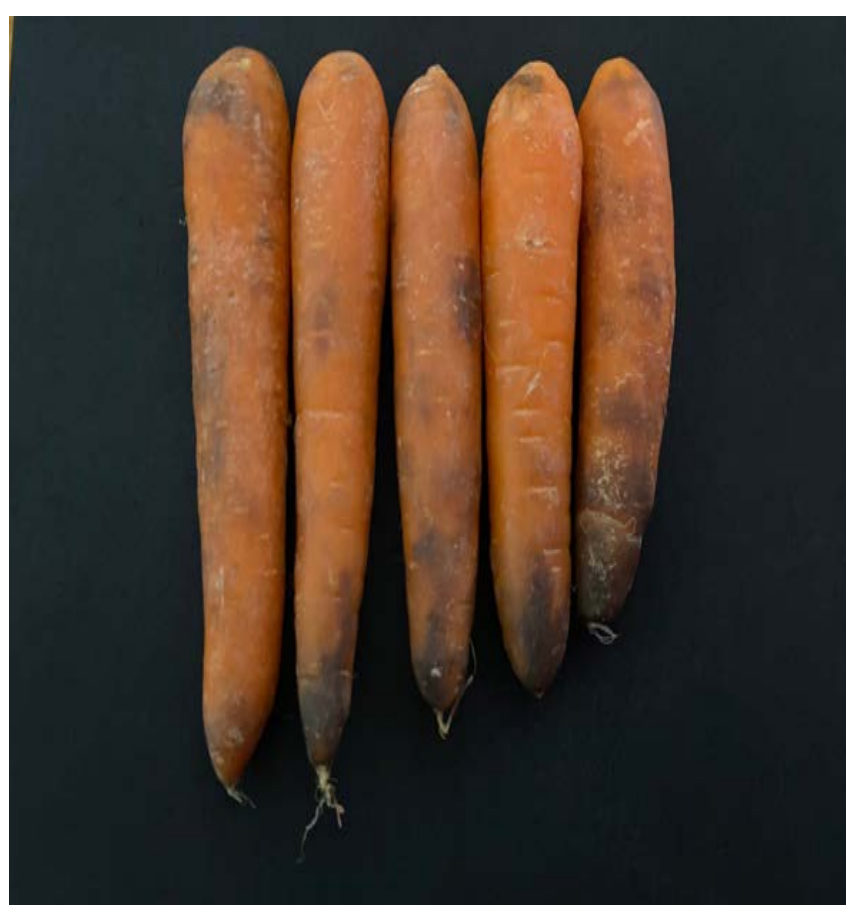

Figura 2. Deterioro de la zanahoria causado por el exceso de transpiración. Imagen tomada en punto de venta. envolturas protectoras que ayuden en la conservación de la raíz (Firpo et al. 2011), será necesario utilizar en los empaques materiales isotérmicos como la poliespuma en mayor proporción que el hule de nylon transparente.

\section{Producción de etileno.}

El etileno es el fitorregulador que acelera la maduración en la mayoría de los vegetales. En la zanahoria, se ha estudiado que este compuesto desempeña un papel importante durante la maduración. Algunas investigaciones confirman que uno de los efectos que produce el etileno en este vegetal es la producción de una molécula llamada isocoumarina, que le confiere sabor amargo (Karkleliené et al. 2012).

La existencia de oxígeno es promovente de la actividad del etileno en los tejidos de zanahoria, y la velocidad de respiración del vegetal está estrechamente relacionada con la producción de etileno (Mahajan et al. 2014); por tal motivo, los empaques deberán impedir la entrada de oxígeno. Blankenship y Dole (2003) indicaron que el 1- metilciclopropeno ayuda a extender la vida de anaquel de productos vegetales y retarda la velocidad de producción de etileno en zanahoria. Por tanto, teniendo en cuenta que no es una sustancia tóxica y que es hidrosoluble y degradable por la amilasa salival, resulta factible su uso en los empaques (Kanayama y Kochetov 2015). En México, el 1- metilciclopropeno no ha sido reportado para el uso en la conservación de zanahoria, por lo que sería importante el desarrollo de investigaciones de este tipo para prolongar la vida de anaquel.

La reducción de temperatura en un tiempo corto es una estrategia que disminuye la producción de 
etileno y, por consiguiente, la aparición de daños visibles. Aunque la tasa de producción de etileno en la zanahoria es baja $\left(0.1 \mu \mathrm{kg}^{-1} \mathrm{~h}^{-1}\right)$ a $20^{\circ} \mathrm{C}$, el incremento de su concentración genera un sabor amargo, debido a la formación de isocumarina. Por lo tanto, las zanahorias no deben almacenarse en conjunto con otros productos que produzcan etileno (González et al. 2010).

\section{Composición bioquímica}

Los compuestos orgánicos en plantas, principalmente los metabolitos secundarios, tienen propiedades nutraceúticas y son conocidos como compuestos bioactivos, fitonutrientes o antioxidantes (Sharma et al. 2012), los cuales intervienen po-
En la zanahoria, los carotenoides aportan la pigmentación naranja que en la mayoría de las variedades caracteriza a la raíz, cuyo rol como protector en el metabolismo ha sido ampliamente descrito (Becaro et al. 2015). Este compuesto y otros como el como el $\beta$-caroteno, el tocoferol y el ácido ascórbico protegen las plantas de condiciones de estrés abiótico como salinidad, sequía y calor (Villeneuve y Geoffriau 2020). El consumo de este vegetal, con alto contenido de $\beta$-caroteno, brinda beneficios diuréticos, confiriendo un adecuado balance de líquidos corporales al organismo que lo consume (Sharma et al. 2012).

El papel del $\beta$-caroteno de la zanahoria en la salud ha sido estudiado hace algunos años (Tanaka et al. 2012), para demostrar que el consumo de este vegetal

Cuadro 3. Principales componentes bioquímicos de la zanahoria.

\begin{tabular}{ll}
\hline Componentes & Valor \\
\hline Agua (\%) & $86.0-89.0$ \\
Proteínas (\%) & $0.7-0.93$ \\
Carbohidratos (\%) & $6.0-10.6$ \\
Fibra cruda (\%) & $1.2-2.8$ \\
Ca (mg $\left.100 \mathrm{~g}^{-1}\right)$ & $34.0-80.0$ \\
Fe (mg $\left.100 \mathrm{~g}^{-1}\right)$ & $0.3-2.2$ \\
$\mathrm{Mg}\left({\left.\mathrm{mg} 100 \mathrm{~g}^{-1}\right)}_{\mathrm{K}\left(\mathrm{mg} 100 \mathrm{~g}^{-1}\right)}\right.$ & $9.0-12.0$ \\
$\mathrm{P}\left(\mathrm{mg} 100 \mathrm{~g}^{-1}\right)$ & $240-320$ \\
Carotenos $\left(\mathrm{mg} 100 \mathrm{~g}^{-1}\right)$ & $25.0-53.0$ \\
Vitamina C (mg $\left.100 \mathrm{~g}^{-1}\right)$ & 5.33 \\
\hline
\end{tabular}

Fuente: Barzee et al. (2019), USDA (2018).

sitivamente en la salud del consumidor, debido a que ayudan a prevenir, tanto la degradación acelerada de macromoléculas y de ácidos nucleicos, como el ataque de radicales libres (Goyal et al. 2019). Dentro de los componentes bioquímicos presentes en la zanahoria, destacan altos contenidos de humedad, carbohidratos, fibras y minerales, que avalan su importancia nutricional (Cuadro 3) (Barzee et al. 2019; USDA 2018).

Algunos estudios señalan que la zanahoria es una importante fuente de compuestos bioactivos, dentro de los cuales se encuentran los fenólicos, poliacetilenos, carotenoides y antocianinas, que previenen el catabolismo de macromoléculas estructurales en el organismo humano y las oxidaciones biológicas (Becaro et al. 2015). coadyuva a prevenir la enfermedad de Alzheimer, y actúa como supresor del virus de inmunodeficiencia humana (Sharma et al. 2012).

La función antioxidante de este compuesto es amplia, y debe resaltarse que es el principal precursor de la vitamina $\mathrm{A}$, involucrada en la síntesis de glicoproteínas, compuestos orgánicos que participan en la síntesis de la púrpura visual, así como en el crecimiento y desarrollo de los huesos (RodríguezConcepción y Stange 2013). Un manejo poscosecha inadecuado, donde existan altas temperaturas y alta humedad relativa, propicia una disminución significativa de los contenidos de carotenos, por lo cual se ve afectada su apariencia y disminuye su función nutricional (Goyal et al. 2019). 
Los vegetales como la zanahoria son una fuente importante de nutrientes que favorecen la buena salud de los consumidores. Además de ser una fuente importante de fibra dietaria, la zanahoria contiene niveles importantes de molibdeno, un mineral raramente encontrado en vegetales. El molibdeno participa en el metabolismo de grasas y carbohidratos, además de facilitar la absorción del hierro (Ilic et al. 2013). También es fuente importante de magnesio y manganeso. El magnesio es un mineral requerido para la formación de huesos, la síntesis de proteínas, la producción de energía, la secreción de insulina y otras funciones vitales (Kim et al. 2010). Por su parte, el manganeso actúa como cofactor de diversas enzimas que participan en el metabolismo de carbohidratos (Dias 2012).

\section{Cosecha}

El momento de cosecha o índice de cosecha de la zanahoria es muy variable, ya que depende del ciclo del cultivar, de las condiciones medioambientales y del tamaño deseado de la raíz. Normalmente, el tiempo de cosecha está determinado por el tamaño máximo o cercano a la longitud del potencial genético productivo de la variedad en cuestión. Se ha estudiado que, entre 70 y 90 días después de la siembra, se obtiene una raíz de hasta $18 \mathrm{~cm}$ de longitud y un diámetro de la corona de $2.5 \mathrm{~cm}$, aunque hay cultivares que requieren de hasta 120 días para alcanzar estas dimensiones. Una zanahoria con una corona de 2.5 centímetros o más, es comercial (Figura 3); sin embargo, algunos mercados prefieren un tipo de raíz con una corona de $2.5 \mathrm{~cm}$ y longitudes entre 14-16 cm para evitar altas con-

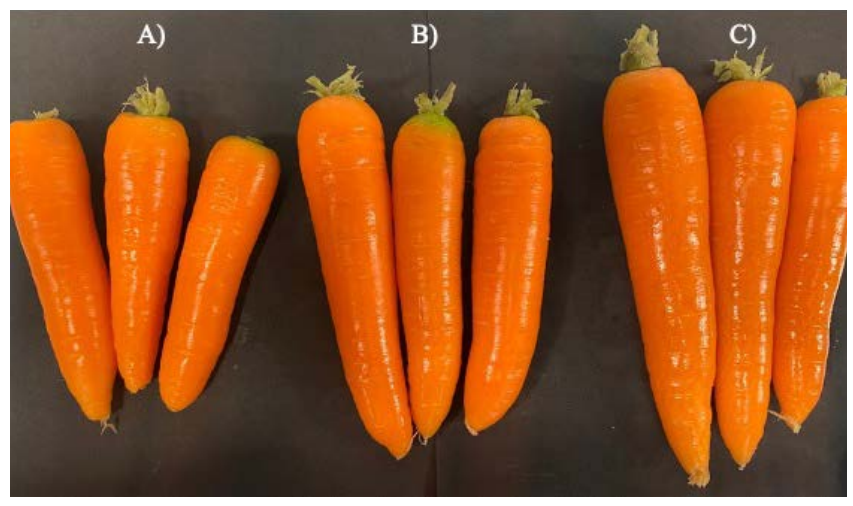

Figura 3. Zanahorias comerciales: A) mercado nacional; B) calidad media; C) jumbo (para exportación). centraciones de celulosa que endurece la raíz y dificulta el consumo mínimamente procesado (Gaviola 2013).

\section{Normas de calidad}

Para la venta de la zanahoria, existen varias normas o lineamientos que establecen las condiciones de comercialización con vistas a la excelencia del producto (Gaviola 2013). En México, la Norma NMX-FF-024-1982 (SCFI 1982) establece las características de calidad que debe cumplir la zanahoria destinada al consumo humano directo. En ella, se describen las características

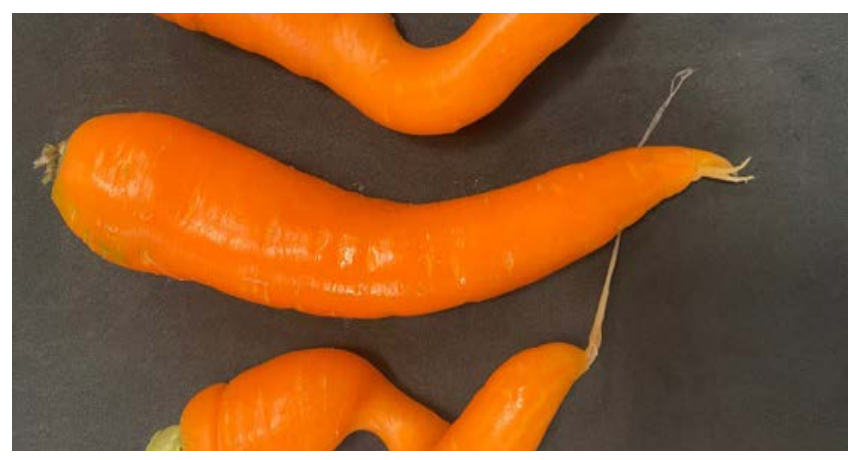

Figura 4. Zanahoria fuera de las categorías de la NMX-FF-024-1982 designada como "No clasificado".

de los tipos y medidas de defectos. En esta NMX se establece que la zanahoria se clasifica en tres grados de calidad en orden descendente: México Extra, México Núm. 1 y Núm. 2. El producto clasificado se designa por su nombre, tamaño y grado de calidad, así que cualquier producto que no entre en alguna de esas categoríassedesignarácomo "Noclasificado" (Figura4).

En general, dentro de las especificaciones sensoriales, una zanahoria debe estar bien desarrollada, entera, sana, fresca, limpia, así como tener una consistencia firme y razonablemente lisa. Asimismo, debe encontrarse libre de descomposición, manchas o pudrición y de defectos de origen mecánico, entomológico, microbiológico, meteorológico y genético-fisiológico, pues éstas son cualidades que avalan la calidad de la raíz (Kyriacou y Rouphael 2018).

Las normas internacionales como la CODEX STAN 320-2015 se aplican para las zanahorias congeladas rápidamente, de la especie $D$. carota, destinada al consumo directo sin una ulterior elaboración, excepto un nuevo envasado, si fuese necesario. No se aplica al producto cuando se indique que se destina a una 
ulterior elaboración o a otros fines industriales (FAO 2015a). Existe, también, la norma internacional CODEX STAN 297-2007, que establece las características de la zanahoria para conserva. Aplica para las zanahorias a las que se les han quitado las hojas, se han lavado y se han envasado con agua u otro medio líquido apropiado como edulcorantes, condimentos y otros ingredientes, y que se han tratado con calor de modo adecuado antes o después de haberse cerrado herméticamente en un recipiente, para evitar su alteración (FAO 2015b).

Otros reglamentos, como los de Estados Unidos, hacen énfasis en las características morfológicas y organolépticas que avalen la alta calidad del producto (USDA 2020). Todos estos reglamentos deben cumplirse, debido a que, al final, el producto que no lo haga será rechazado, y el productor tendrá pérdidas económicas considerables.

\section{Desórdenes fisiológicos que afectan la vida de anaquel de la zanahoria}

En la actualidad, hay una gran variedad de métodos para conservar vegetales $y$, en particular, raíces (Dussán-Sarria et al. 2015); sin embargo, si no se tienen las técnicas adecuadas para una correcta conservación, pueden causar trastornos fisiológicos poscosecha $y$, posteriormente, daños patológicos, lo que ocasionaría pérdidas importantes (Wu 2010). En zanahoria, hay algunos desordenes fisiológicos que son desencadenados por un mal manejo poscosecha del producto, lo que acarrea grandes pérdidas económicas (Rogers et al. 2011).

El síndrome de hombros verdes (Figura 5) es causado por la exposición a la luz solar de la parte

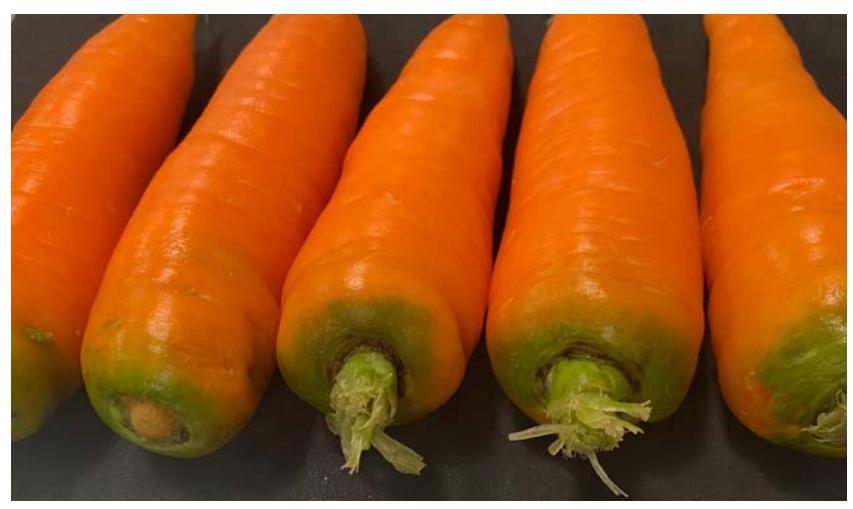

Figura 5. Síndrome de hombros verdes en zanahoria. superior de la raíz, por lo que su control se logra haciendo un pequeño aporque que cubra por completo la raíz de la zanahoria (Han et al. 2013). También se ha observado la tendencia a generar síndrome de hombro verde cuando la raíz cosechada es expuesta por más de cuatro horas a la radiación solar directa (Gruszecki et al. 2015).

La exposición de la raíz cosechada a temperaturas variadas también puede generar desordenes fisiológicos. El congelamiento causa un colapso inmediato de tejidos y la pérdida total del producto (Arkoub-Djermoune et al. 2020). Al exponerse a temperaturas entre 5 y $15^{\circ} \mathrm{C}$ ocurren oscurecimientos internos y en la superficie (pardeamiento), algunos hundimientos en la corteza, coloración disforme, aparición de hongos y pudriciones; sin embargo, la exposición de la raíz cosechada al calor puede causar ablandamiento excesivo y desecación (Dussán-Sarria et al. 2015). La temperatura es una variable climática importante al momento de la cosecha. Generalmente, se debe cosechar en horas de la tarde, aunque la preferencia por parte de los productores siempre es hacerlo por la mañana (Soldado-Soldado et al. 2018).

\section{Desórdenes patológicos}

Algunas enfermedades que afectan la zanahoria son la podredumbre blanda bacteriana, causada por Erwinia carotovora subsp. carotovora (Jones) Bergey et al. y E. carotovora subsp. atroseptica (Van Hall) Dye. Esta enfermedad es fuertemente destructora de hortalizas almacenadas y en tránsito, y la sintomatología característica es una pudrición blanda y hedionda (Marquez-Villavicencio et al. 2011). Generalmente, los desórdenes patológicos poscosecha son debidos al mal manejo de plagas y enfermedades. Muchos productores prefieren prestar atención a estas labores para evitar desinfecciones a base de soluciones de hipoclorito de sodio y otros bactericidas usados durante el manejo poscosecha (Seo et al. 2014), los cuales, además de encarecer los costos, pueden llegar a modificar la superficie de la raíz y, por consiguiente, disminuir su apariencia física en los anaqueles (Jayaraj et al. 2008).

Es común que el producto sufra daños mecánicos (golpes, raspaduras, magulladuras, puntas quebradas, rajaduras) durante la eliminación de hojas, el lavado 


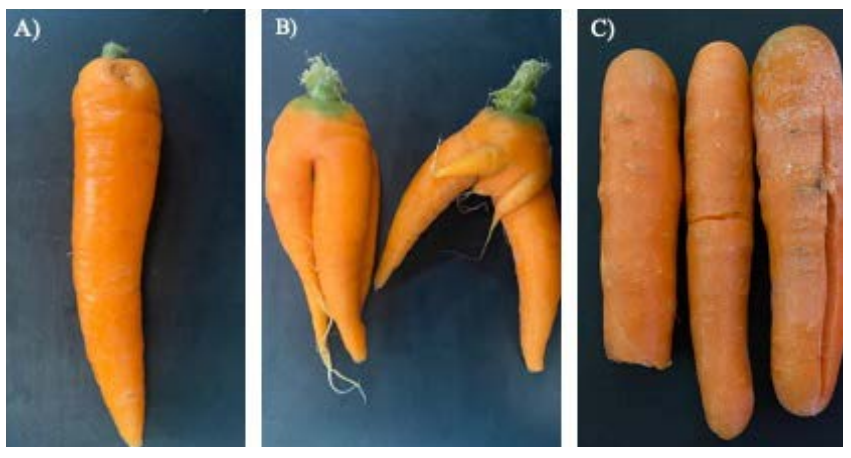

Figura 6. Zanahorias fuera de calidad por daños mecánicos A) cicatrices; B) deformaciones; C) quebraduras o rajaduras.

y el transporte. Además, existen deformaciones ocasionadas por pedregosidad en el suelo o suelos mal preparados (Figura 6), que propician la obtención de un producto fuera de los estándares de calidad; asimismo, los daños aumentan la pérdida de agua y originan una zanahoria flácida o con susceptibilidad a la presencia de hongos.

Entre los hongos que afectan significativamente la calidad poscosecha de esta hortaliza se encuentra Thielaviopsis thielavioides (Peyr.) Paulin, Harr. \& McNew, que causa podredumbre poscosecha, lo que ocasiona pérdidas económicas importantes ( $\mathrm{Xu}$ et al. 2020). En la investigación efectuada, sin embargo, no se encontraron referencias científicas sobre su presencia en México.

\section{La refrigeración durante la conservación de la zanahoria}

Durante la refrigeración para la conservación y traslado de la zanahoria, se deben tener en cuenta dos elementos: 1) disminuir la respiración, la transpiración y la producción de etileno, y 2) evitar el punto de congelación, ya que las pérdidas por congelación son irreversibles, y los daños, visibles en cuanto se produce el proceso de descongelación (Escobar et al. 2014). Los equipos de refrigeración deberán mantener temperaturas entre $10^{\circ} \mathrm{C}$ y $14^{\circ} \mathrm{C}$, y humedad relativa superior a 90 por ciento (Barzee et al. 2019) para propiciar baja respiración, la transpiración y síntesis de etileno, y, al mismo tiempo, evitar el congelamiento celular (Goyal et al. 2019).

Durango et al. (2006) establecieron que las mayores pérdidas en zanahoria son por alteraciones en la respiración celular, con resultados en los que se daba una disminución de 15 por ciento de la turgencia a las 14 horas de efectuada la cosecha, cuando se usaban empaques tradicionales, mientras que el uso de polietileno negro retardaba la pérdida de turgencia a las $168 \mathrm{~h}$. Dentro de las diferentes tecnologías desarrolladas, para el control de la respiración poscosecha es la adecuada refrigeración (máximo $14^{\circ} \mathrm{C}$ y humedad relativa superior a 90\%) (Pushkala et al. 2012).

Para la correcta refrigeración de la zanahoria es necesario llevar a cabo un preenfriamiento, con la finalidad de extraer rápidamente la temperatura de campo del vegetal recién cosechado, previo a su procesamiento, almacenamiento o transporte refrigerado, y así maximizar la vida poscosecha del producto (FAO 2013).

\section{Atmósferas modificadas}

La intensidad respiratoria, la humedad relativa, temperatura y permeabilidad del empaque determinan las condiciones de concentración de $\mathrm{O}_{2}$ y $\mathrm{CO}_{2}$ dentro de dicho embalaje (Amanatidou et al. 2000). Cuando se aplican concentraciones de 5 a 10 por ciento de $\mathrm{CO}_{2} \mathrm{y}$ de 2 a 5 por ciento de $\mathrm{O}_{2}$ se mantienen las características de la raíz hasta 240 días. Sin embargo, se recomienda una combinación de $50 \%$ de $\mathrm{O}_{2}$ y $30 \%$ de $\mathrm{CO}_{2}$ para garantizar una vida de anaquel entre 5 y 7 días. En estas condiciones, se conservan $100 \%$ de las características iniciales de la zanahoria cosechada, en comparación con el almacenamiento en aire (Pinto et al. 2017). Estos resultados evidencian que para la conservación de la zanahoria es importante previamente analizar la relación oferta-demanda, pues en ocasiones no es necesario disminuir las concentraciones de $\mathrm{CO}_{2}$ y $\mathrm{O}_{2}$ cuando la oferta se presenta en un tiempo considerablemente corto.

\section{Recubrimientos comestibles}

Hace algún tiempo se innovaron recubrimientos orgánicos o películas comestibles en empaques bioactivos para inhibir o retardar el crecimiento de microorganismos (Costa et al. 2012), como sucesores al uso de diversos compuestos como polisacáridos, glucoproteínas y glucolípidos (Durango et al. 2006). Actualmente, la tendencia es usar ácidos orgánicos, entre ellos: el ascórbico, el propiónico, el benzoico, y el 
elágico (Rojas et al. 2015), y oxisales como el benzoato de sodio, que ofrecen un mayor control de la actividad microbiana (Escobar et al. 2014).

Pushkala et al. (2012) elaboraron un recubrimiento a base de un polisacárido obtenido del exoesqueleto de crustáceos, el quitosano, el cual tiene propiedades antimicrobianas y antifúngicas. Otro ejemplo de aplicación de recubrimiento de zanahoria para su conservación fue elaborado a base de alginato de sodio, a partir de algas marinas de la familia Phaephyceae (García et al. 2011). Por último, Costa et al. (2012) elaboraron un recubrimiento con alginato de calcio en zanahoria al igual que el estudio de Mastromatteo et al. (2012). Sin embargo, estos recubrimientos no han tenido mucho éxito, debido al alto costo de los compuestos orgánicos empleados (Sillani y Nassivera 2015).

\section{Productos mínimamente procesados}

Los productos mínimamente procesados (PMP), son aquellos que han sido convenientemente pelados, cortados, empacados en fresco y listos para el consumo, con la ventaja de no requerir algún otro proceso antes de su consumo (Dussán-Sarria et al. 2014). Los vegetales frescos mínimamente procesados han tenido un crecimiento en los últimos años, de acuerdo con las nuevas tendencias de producción orgánica y el desarrollo de tecnologías en los países industrializados, pues el actual ritmo de vida, en el que los consumidores tienen un escaso tiempo para preparar comidas equilibradas, ha provocado la demanda de productos vegetales naturales, frescos, saludables y listos para consumir (Glowacz y Rees 2016).

El uso de ozono durante la conservación de PMP se considera una alternativa viable en la industria alimentaria, debido a su potencial como germicida y como agente oxidante; por esta razón, resulta efectivo contra varios tipos de microorganismos patógenos y mejora la calidad de los alimentos. El agua del proceso de lavado con el empleo del ozono puede ser reusada, con lo que disminuyen los costos de operación y el impacto ambiental de los efluentes (Bataller-Venta et al. 2010). Aunque en México no existen reportes sobre el uso del ozono en el manejo poscosecha de la zanahoria a escala comercial, las experiencias efectuadas experimentalmente demuestran que es una opción sustentable en la poscosecha de frutas y hortalizas, en general, y de zanahoria, en particular (Ramos et al. 2013).

Las zanahorias mínimamente procesadas también pueden presentar deterioro (cambios físicos) durante su almacenamiento, debido a tres fenómenos: 1) respuesta fisiológica a los factores de estrés, 2) deshidratación superficial y 3) crecimiento microbiano (Becaro et al. 2015). Dichos factores pueden generar cambios químicos, entre ellos, la síntesis de compuestos fenólicos esterificados a la lignina, que endurecen o lignifican la raíz cosechada (Kehr y Borquez 2010). Estos factores también pueden propiciar el aumento de la concentración de peróxido de hidrógeno. La formación de lignina es medida por el porcentaje de blanqueamiento; estos procesos disminuyen la calidad sensorial y, por consiguiente, la aceptabilidad de los consumidores (Becaro et al. 2015). Debido a lo anterior, la refrigeración tiene gran importancia para evitar la pérdida de la apariencia física, la síntesis de etileno y la presencia de agentes patógenos; lo anterior deberá complementarse con un adecuado empaque para su conservación, traslado y permanencia en los anaqueles antes de ser consumidos.

\section{Conclusiones}

En México, las investigaciones sobre la fisiología poscosecha de la zanahoria son escasas, aunque es un vegetal que se produce en varios estados. Sus propiedades bioquímicas y nutraceúticas confirman la importancia que presenta para la salud humana.

El manejo poscosecha inadecuado puede propiciar la pérdida de sus cualidades organolépticas y la aparición de enfermedades bacterianas y fúngicas, que merman su calidad y vida de anaquel. Para evitar estos desórdenes fisiológicos y patológicos es preferible una temperatura de refrigerado de $14^{\circ} \mathrm{C}$ y una humedad relativa superior a $90 \%$ durante su almacenamiento y traslado hacia el punto de venta. 


\section{Literatura Citada}

Acevedo A. 2018. Identificación morfológica y molecular de cepas del hongo Alternaria dauci patógeno de la zanahoria (Daucus carota L.). Aporte Santiaguino 11: 21-30. https://doi.org/10.32911/as.2018.v11.n1.453

Amanatidou A, Slump RA, Gorris LGM, Smid EJ. 2000. High oxygen and high carbon dioxide modified atmospheres for shelf-life extension of minimally processed carrot. Journal of Food Science 65: 61-66. https://doi. org/10.1111/j.1365-2621.2000.tb15956.x

Arkoub-Djermoune L, Louaileche H, Benmeziane F, Madani K, Boulekbache-Makhlouf L. 2020. Impact of refrigerated storage on the bioactive compounds and antioxidant capacity of two Algerian carrot varieties (Daucus carota L.). Acta Universitatis Sapientiae, Alimentaria 13: 5-31. https://doi.org/10.2478/ ausal-2020-0001

Barzee TJ, El-Mashad HM, Zhang R, Pan Z. 2019. Carrots. En: Pan Z, Zhang R, Zicari S, editores. Integrated Processing Technologies for Food and Agricultural By-Products. San Diego, Elsevier. P. 297-330.

Bataller-Venta M, Cruz-Broche SS, García-Pérez MA. 2010. El ozono: una alternativa sustentable en el tratamiento poscosecha de frutas y hortalizas. Revista CENIC Ciencias Biológicas 41: 155-164.

Becaro AA, Puti FC, Panosso AR, Gern JC, Brandao HM, Correa DS, Ferreira MD. 2015. Postharvest quality of fresh-cut carrots packaged in plastic films containing silver nanoparticles. Food and Bioprocess Technology 9: 637-649. https://doi.org/10.1007/s11947-015-1656-z

Blankenship SM, Dole JM. 2003. 1-Methylcyclopropene: A review. Postharvest Biology and Technology 28: 1-25. https://doi.org/10.1016/S0925-5214(02)00246-6

[CEDRSSA] Centro de Estudios para el Desarrollo Rural Sustentable y la Soberanía Alimentaria. [internet]. 2020. Análisis de la producción y consumo de hortalizas. Ciudad de México. [citado 2021 enero 3]. Disponible en: http://www.cedrssa.gob.mx/post_n-annolisis_-n-_de_la_-_n-produccinin_-n-_y_--n-consumo_-n-_de_-n-hortalizas_-n.htm

Costa C, Conte A, Buonocore GG, Lavorgna M, Del Nobile MA. 2012. Calcium-alginate coating loaded with silver-montmorillonite nanoparticles to prolong the shelf-life of fresh-cut carrots. Food Research International 48: 164-169. https://doi.org/10.1016/j. foodres.2012.03.001
Dias JS. 2012. Major classes of phytonutriceuticals in vegetables and health benefits: A review. Journal of Nutritional Therapeutics 1:31-62.

Dong R, Yu Q, Liao W, Liu W, Liu S, He Z, Hu X, Chen Y, Xie J, Nie S, Xie M. 2021. Composition of bound polyphenols from carrot dietary fiber and its in vivo and in vitro antioxidant activity. Food Chemistry 339: 127879. https://doi.org/10.1016/j.foodchem.2020.127879

Durango AM, Soares NFF, Andrade NJ. 2006. Microbiological evaluation of an edible antimicrobial coating on minimally processed carrots. Food Control 17: 336-341. https://doi.org/10.1016/j.foodcont.2004.10.024

Dussán-Sarria S, Torres-León C, Hleap-Zapata JI. 2014. Efecto de un recubrimiento comestible y de diferentes empaques durante el almacenamiento refrigerado de mango Tommy Atkins mínimamente procesado. Información Tecnológica 25: 123-130. https://doi. org/10.4067/S0718-07642014000400014

Dussán-Sarria S, Garcia-Mogollon CA, Gutiérrez-Guzmán N. 2015. Cambios físico-químicos y sensoriales producidos por el tipo de corte y empaque en zanahoria (Daucus carota L.) mínimamente procesada. Información Tecnológica 26: 63-70. https://doi.org/10.4067/ S0718-07642015000300010

Escobar A, Márquez CJ, Restrepo CE, Cano JA, Patiño JH. 2014. Aplicación de tratamiento térmico, recubrimiento comestible y baño químico con tratamientos poscosecha para la conservación de hortalizas mínimamente procesadas. Acta Agronómica 63: 1-10. https://doi. org/10.15446/acag.v63n1.40149

[FAO] Organización de las Naciones Unidas para la Alimentación y la Agricultura. [internet]. 2013. Manual para la preparación y venta de frutas y hortalizas de la Organización de las Naciones Unidas para la Agricultura y la Alimentación. Boletín de servicios agrícolas de la FAO. [citado 2019 marzo 17]. Disponible en: http://www.fao.org/docrep/006/ y4893s/y4893s00.htm

[FAO] Organización de las Naciones Unidas para la Alimentación y la Agricultura. [internet]. 2015a. Norma para hortalizas congeladas rápidamente CODEX STAN 320-2015. [citado 2019 mayo 19]. Disponible en: www.fao.org/input/download/standards/13928/ CXS_320s_2015.pdf

[FAO] Organización de las Naciones Unidas para la Alimentación y la Agricultura. [internet]. 2015b. Norma para algunas hortalizas en conserva CODEX 
STAN 297-2007. [citado 2019 mayo 19]. Disponible en: www.fao.org/input/download/standards/11253/ CXS_297s_2015.pdf

Firpo I, Rotondo R, Ferratto J, Grasso R, Trevisan A, Rodriguez M. 2011. Incidencia del ambiente y la revitalización en las pérdidas poscosecha de hortalizas de hoja, fruto y raíz. Ciencias Agronómicas 20: 7-13.

García MA, Ventosa M, Díaz R, Casariego A. 2011. Efecto de coberturas de alginato de sodio enriquecidas con Aloe vera en la calidad de la zanahoria mínimamente procesada. Ciencia y Tecnología de Alimentos 21: 62-67.

Gaviola J. 2013. Manual de producción de zanahoria. Instituto Nacional de Tecnología Agropecuaria. Buenos Aires, Argentina.

Glowacz M, Rees D. 2016. The practicality of using ozone with fruit and vegetables. Journal of the Science of Food and Agriculture 96: 4637-4643. https://doi. org/10.1002/jsfa.7763

González G, Galviz J, Florez A. 2010. Manual de zanahoria minimamente procesada, variedades Chantenay y Baby. Fundación Universitaria Agraria de Colombia. Bogotá, Colombia.

Goyal M, Joy P, Suleria H. 2019. Plant Secondary Metabolites for Human Health. Taylor \& Francis Group. Florida, USA.

Gruszecki R, Borowy A, Salata A, Zawislak G. 2015. Effect of living mulch and linuron on weed and yield of carrot under ridge cultivation. Acta Scientiarum Polonorum Hortorum Cultus 14: 67-82.

Han Z, Deng L, Xu Y, Feng Y, Geng Q, Xiong K. 2013. Image processing method for detection of carrot green-shoulder, fibrous roots and surface cracks. Transactions of the Chinese Society of Agricultural Engineering 29: 156161. https://doi.org/10.3969/j.issn.1002-6819.2013.09.020

Hernández RM, Blanco DJ. 2015. Evaluación de polvos de zanahoria obtenidos por deshidratación por aire forzado a diferentes temperaturas. IDESIA 33: 75-80. https:// doi.org/10.4067/S0718-34292015000400010

Ilic Z, Šunic L, Barac S, Stanojevic L, Cvetkovic D, Marinkovic D. 2013. Effect of postharvest treatments and storage conditions on quality parameters of carrots. Journal of Agricultural Science 5: 100-106. https:// doi.org/10.5539/jas.v5n5p100

Jayaraj J, Wan A, Rahman M, Punja ZK. 2008. Seaweed extract reduces foliar fungal diseases on carrot. Crop Protection 27: 1360-1366. https://doi.org/10.1016/j. cropro.2008.05.005
Kanayama Y, Kochetov A. 2015. Abiotic Stress Biology in Horticultural Plants. Springer. Miyagi, Japón.

Karkleliené R, Radzevičius A, Dambrauskiené E, Surviliené E, Bobinas Č, Dushovskiené L, Kavaliauskaité D, Bundiniené O. 2012. Root yield, quality and disease resistance of organically grown carrot (Daucus sativus Röhl.) hybrids and cultivars. Zemdirbyste Agriculture 99: 393-398.

Karnjanawipagul R, Nittayanuntawech W, Rojsanga P, Suntornsuk L. 2010. Analysis of B-carotene in carrot by spectrophotometry. Mahidol University Journal of Pharmaceutical Science 37: 8-16.

Kehr E, Borquez C. 2010. La zanahoria como una hortaliza apta para el procesamiento agroindustrial. Tierra Adentro 88: 17-19.

Kim DJ, Xun P, Liu K, Loria C, Yokota K, Jacobs DR, He K. 2010. Magnesium intake in relation to systemic inflammation, insulin resistance, and the incidence of diabetes. Diabetes Care 33: 2604-2610. https://doi. org/10.2337/dc10-0994

Kyriacou M, Rouphael Y. 2018. Towards a new definition of quality for fresh fruits and vegetables. Scientia Horticulturae 234: 463-469. https://doi.org/10.1016/j. scienta.2017.09.046

Mahajan P, Caleb OJ, Singh Z, Watkins CB, Geyer M. 2014. Postharvest treatments of fresh produce. Philosophical Transactions of the Royal Society A 372: 20130309. https://doi.org/10.1098/rsta.2013.0309

Marquez-Villavicencio MP, Groves RL, Charkowski AO. 2011. Soft rot disease severity is affected by potato physiology and Pectobacterium taxa. Plant Disease 95: 232-241. https://doi.org/10.1094/PDIS-07-10-0526

Martínez-González ME, Balois-Morales R, Alia-Tejacal I, Cortes-Cruz MA, Palomino-Hermosillo AY, LópezGuzmán GG. 2017. Postcosecha de frutos: maduración y cambios bioquímicos. Revista Mexicana de Ciencias Agrícolas (Núm. Esp. 19): 4075-4087. https://doi. org/10.29312/remexca.v0i19.674

Mastromatteo M, Conte A, Del Nobile MA. 2012. Packaging strategies to prolong the shelf life of fresh carrots (Daucus carota L.). Innovative Food Science \& Emerging Technologies 13: 215-220. https://doi. org/10.1016/j.ifset.2011.10.010

Nesha R, Siddiqui ZA. 2013. Interactions of Pectobacterium carotovorum pv. carotovorum, Xanthomonas campestris pv. carotae and Meloidogyne javanica on the disease complex of carrot. International Journal of Vegetable Science 19: 
403-411. https://doi.org/10.1080/19315260.2012.744379

Pandey N, Rijal S, Adhikari H, Bhantana B, Adhikar M. 2020. Production economics and determinants of carrot (Daucus carota L.) production in Chitwan, Nepal. International Journal of Social Sciencies and Management 7: 234-241. https://doi.org/10.3126/ijssm. v7i4.32473

Pinto N, de la Vega JC, Cañarejo M. 2017. Utilización del método de conservación bajo atmósferas controladas en frutas y hortalizas. Agroindustrial Science 6: 231-238.

Pushkala R, Parvathy KR, Srividya N. 2012. Chitosan powder coating, a novel simple technique for enhancement of shelf life quality of carrot shreds stored in macro perforated LDPE packs. Innovative Food Science \& Emerging Technologies 16: 11-20. https://doi. org/10.1016/j.ifset.2012.03.003

Ramos B, Miller FA, Brandão TRS, Teixeira P, Silva CLM. 2013. Fresh fruits and vegetables-An overview on applied methodologies to improve its quality and safety. Innovative Food Science \& Emerging Technologies 20: 1-15. https://doi.org/10.1016/j.ifset.2013.07.002

Rodríguez-Casado A. 2016. The health potential of fruits and vegetables phytochemicals: Notable examples. Critical Reviews in Food Science and Nutrition 56: 10971107. https://doi.org/10.1080/10408398.2012.755149

Rodríguez-Concepción M, Stange C. 2013. Biosynthesis of carotenoids in carrot: An underground story comes to light. Archives of Biochemistry and Biophysics 539: 110116. https://doi.org/10.1016/j.abb.2013.07.009

Rogers P, Stevenson W, Wyman J, Frost K, Groves R. 2011. IPM Perspectives for Carrot Foliar Disease in Wisconsin. University of Wisconsin. Madison, USA.

Rojas R, Vicente AA, Aguilar CN. 2015. Advances in preservation of fruits and vegetables with bioactive coatings. Boletim do Centro de Pesquisa de Processamento de Alimentos 33: 45-58. https://doi.org/10.5380/cep. v33i2.46231

[SCFI] Secretaría de Comercio y Fomento Industrial. [internet]. 1982. Norma mexicana sobre productos alimenticios no industrializados para uso humano. Tubérculo. Zanahoria (Daucus carota). [citado 2019 marzo 22]. Disponible en: https://www.colpos.mx/bancodenormas/ nmexicanas/NMX-FF-024-1982.PDF

Seo Y, Park J, Kim YS, Park Y, Kim YH. 2014. Screening and histopathological characterization of Korean carrot lines for resistance to the root-knot nematode Meloidogyne incognita. The Plant Pathology Journal
20: 75-81. https://doi.org/10.5423/PPJ.OA.08.2013.0082 Sharma KD, Karki S, Thakur NS, Attri S. 2012. Chemical composition, functional properties and processing of carrot-a review. Journal of Food Science Technology 49: 22-32. https://doi.org/10.1007/s13197-011-0310-7

[SIAP] Servicio de Información Agroalimentaria y Pesquera. [internet]. 2018. Anuario estadístico de la producción agrícola. [citado 2019 mayo 19]. Disponible en: https://nube.siap.gob.mx/cierreagricola/

Sillani S, Nassivera F. 2015. Consumer behavior in choice of minimally processed vegetables and implications for marketing strategies. Trends in Food \& Technology 46: 339-345. https://doi.org/10.1016/j.tifs.2015.07.004

Singh D, Sharma R. 2018. Postharvest diseases or fruits vegetables and their management. En: Wasim SM, editor. Postharvest Disinfection in Fruits and Vegetables. Sabour, India. Academic Press. P. 1-52. https://doi. org/10.1016/B978-0-12-812698-1.00001-7

Soldado-Soldado G, Valiño E, Fernández-Páramo L, Viafara-Banguera D, Huilca-Álvarez W, Morocho-León E, Ortíz-Nacaza P, Riofrío-Carrión A, Andrade-Yucailla V. 2018. Efecto de los tiempos de recolección post cosecha de Daucus carota sobre la composición química del tubérculo, hojas y tallos como sustrato para ensilaje. Revista Ecuatoriana de Investigaciones Agropecuaria 2: 20-24. https://doi.org/10.31164/reiagro.v2n1.4

Stolarczyk J, Janick J. 2011. Carrot: History and iconography. Chronica Horticulturae 51: 13-18.

Tanaka T, Shnimizu M, Moriwaki H. 2012. Cancer Chemoprevention by carotenoids. Molecules 17: 32023242. https://doi.org/10.3390/molecules17033202

Turati F, Rossi M, Pelucchi C, Levi F, La Vecchia C. 2015. Fruit and vegetables and cancer risk: A review of southern European studies. British Journal of Nutrition 113: S102-S103. https://doi.org/10.1017/S0007114515000148

[USDA] United States Department of Agriculture. [internet]. 2018. 11124 Carrots, raw. National Nutrient Database for Standard Reference Legacy Release. Agricultural Research Service. [citado 2020 diciembre 23]. Disponible en: https://ndb.nal.usda.gov/ndb/ search/list.

[USDA] United States Department of Agriculture. [internet]. 2020. United States Standards for Grades of Topped Carrots. [citado 2020 diciembre 23]. Disponible en: https://www.ams.usda.gov/sites/default/files/media/ToppedCarrotsStandards.pdf

Villeneuve F, Geoffriau E. 2020. Carrot physiological dis- 
orders and crop adaptation to stress. En: Emmanuel G, Philipp WS, editores. Carrots in Related Apiaceae Crops. Wallingford, Cabi. P. 156-170.

Wu C. 2010. An overview of postharvest biology and technology of fruits and vegetables. Proceedings of 2010 AARDO Workshop. Taiwan Agricultural Research Institute. Taiwan, China.

Xu K, Li J, Yang X, Zhang R, Li X, Xie M, Huang Q. 2020. Postharvest rot on carrot caused by Ceratocystis fimbriata and Chalaropsis thielavioides (=Thielaviopsis thielavioides) in China. Journal of General Plant Pathology 86: 322325. https://doi.org/10.1007/s10327-020-00919-1

Yildiz F, Wiley R. 2017. Minimally Processed Refrigerated Fruits and Vegetables. Springer Nature. Adana, Turquía. 